\title{
A Study on the Response of the Rehabilitation Lower Device using Sliding Mode Controller
}

\author{
Dao Minh Duc \\ Faculty of Engineering and Technology \\ Pham Van Dong University \\ Quang Ngai, Vietnam \\ dmduc@pdu.edu.vn
}

\author{
Tran Xuan Tuy \\ Faculty of Mechanical Engineering \\ Da Nang University of Science and Technology \\ Da Nang, Vietnam \\ tranxuantuy@yahoo.fr
}

\author{
Pham Dang Phuoc \\ Faculty of Engineering and Technology \\ Pham Van Dong University \\ Quang Ngai, Vietnam \\ pdphuoc@pdu.edu.vn
}

\begin{abstract}
This paper presents the application of the sliding mode controller to simulate the control of a 3 Degree of Freedom (DoF) robot model that supports lower extremity rehabilitation exercises for stroke patients. The $3 \mathrm{DoF}$ robot model has an established dynamic differential equation for each joint. This paper studied the response of links of the research model with a sliding mode controller proposed as the control solution. Matlab software was used in simulations of ankle, knee, and hip joints' drive for 2 cases, without and with load. Compared with other studies, the research results show that the sliding controller results in slight angular errors when applied to the research model, while the torque of the joints remains low. This result is the basis for calculating and selecting parameters for the actuator when manufacturing the actual equipment.
\end{abstract}

Keywords-sliding mode controller; ankle; knee; hip; rehabilitation

\section{INTRODUCTION}

According to the World Health Organization (WHO), worldwide, each year 15 million people suffer a stroke, of which 5 million die and 5 million become permanently disabled [1]. Vietnam has a population of more than 90 million people and the respective number of newly infected people is 225,000 with 540,720 surviving and 117,900 people dying annually [2]. A cerebrovascular accident will leave severe consequences to some patients. The survivors of strokes commonly are not able to perform daily activities such as dressing, eating, and bathing. In recent years, different researches have been done to develop robotic devices for rehabilitation, especially for the neurorehabilitation of poststroke patients [3]. Rehabilitation after stroke exercise for patients has become an urgent major social issue. Currently, in hospitals and rehabilitation centers, the demand for automatic rehabilitation equipment has increased significantly. Robotic rehabilitation has some benefits over conventional manual rehabilitation, such as:
- Robots can efficiently perform repetitive therapeutic exercises.

- Employing different sensors in robotic systems enables either accurate exertion of torques/forces or precise quantitative evaluation of the patient's recovery during the rehabilitation program.

- Rehabilitation strategies using robotic systems provide simultaneous and coordinated actuation of the patient's joints.

In this study a controller is designed for an exoskeleton rehabilitation robot, performing different passive, assistive, and resistive therapeutic exercises [4]. Research and manufacturing of equipment to support rehabilitation exercises for stroke patients have achieved certain achievements [5-6]. To successfully research and manufacture assistive devices for stroke patients, calculating and simulating the device's response is the first thing before manufacturing. Thanks to the development of computers over the years, simulation has become an indispensable tool for synthesizing control laws in mechanical systems, allowing the verification of the behavior of control laws on a virtual model of an automatic system called simulation model.

Currently, the PID controller is widely applied [7], especially in robot control, to support rehabilitation for stroke patients with the advantages of simple and easy to control structure [8]. The disadvantage is that it is difficult to find the $K_{p}, K_{\mathrm{j}}, K_{d}$ coefficients when the load changes. In addition, modern control theory is increasingly widely applied [9]. The use of a fuzzy controller to control the device has resulted [10]. Authors in [11] used a combination of fuzzy and PID controllers to control their research equipment. The advantage of this controller is that it optimizes the search for control parameters and can automatically adjust the control parameters of other controllers when combined with a PID controller. 
However, for more accuracy, it needs more fuzzy grades, which increases the rules, the lower speed, and the long run time of the system exponentially. In this paper, we introduce the sliding mode controller for the research model. This controller has the advantage of being less affected by load changes. However, it is necessary to overcome the chattering phenomenon of the controller.

\section{DYNAMIC EQUATIONS OF THE RESEARCH MODEL}

In this study, a drive model of lower extremity joints was established with 3 Degrees of Freedom (DoFs) for hip, knee, and ankle joints flexion/extension exercises. The range of motion for hip, knee, and ankle joint was $0-90^{\circ},-135^{\circ}-0^{\circ}, 40^{\circ}$ $120^{\circ}$ respectively [12]. The mechanism that drives the joints is a linear actuator with the advantage of large load capacity and self-braking to protect the patient during the exercise. This paper introduces a research model to contribute to the creation of lower limb rehabilitation equipment. The hip, knee, and ankle joints are reduced to a system with only $3 \mathrm{DoFs}$, and the dynamic computational model for the research model is presented in Figure 1. In the model, link 1 is for the hip joint, link 2 is for the knee joint, and link 3 is for the ankle joint. Setting up the dynamic equation for each link is an essential factor to calculate and simulate the motion of the joints, from which it is possible to calculate the design of the mechanical structure and the controller so that it is suitable for research models.

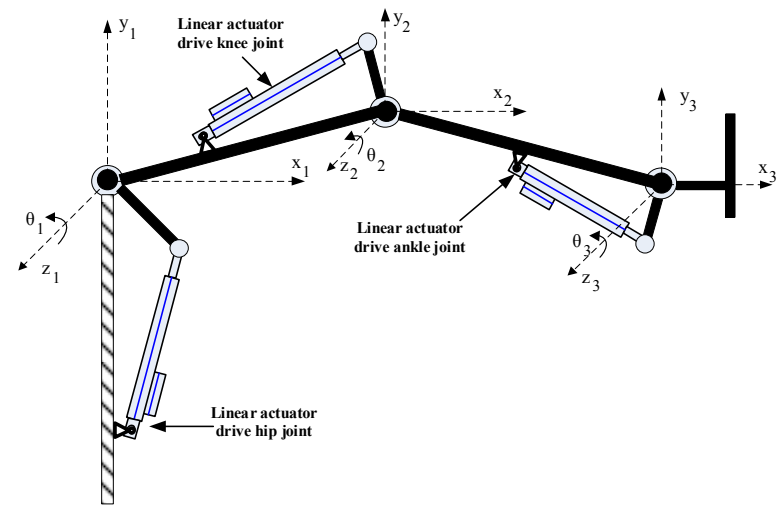

Fig. 1. The research model.

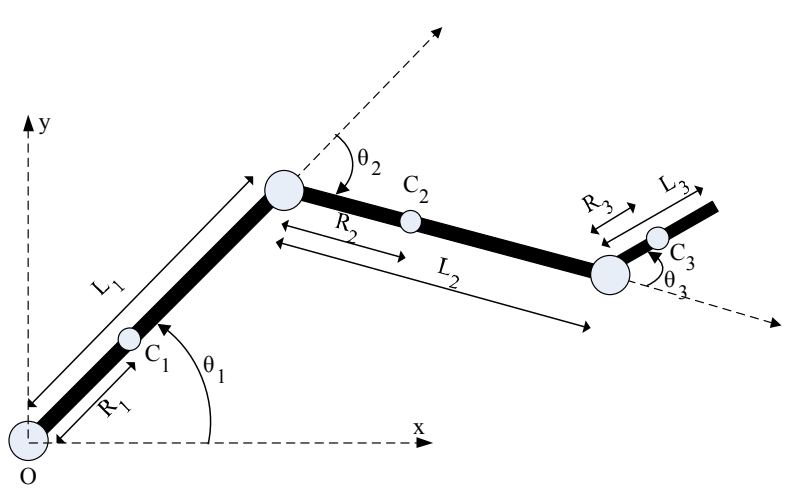

Fig. 2. Dynamic mathematical model.
To establish the dynamic equation of the device [13-14], we conduct a dynamic analysis of the joints. The diagram of the dynamic analysis of the research model is shown in Figure 2. The rotation angles of the joints are shown with the rotation angles of the hip joint is $\theta_{1}$, the knee joint is $\theta_{2}$, and the ankle joint is $\theta_{3}$. To simplify the establishment of kinematic equations of joints, we reduce the mass of each link to the centroid of each link.

The dynamic equation of the model is described as follows:

$$
\begin{aligned}
& D_{n}\left(\theta_{n}\right) \ddot{q}+H_{n}\left(\theta_{n}, \dot{\theta}_{n}\right)+G_{n}\left(\theta_{n}\right)=\tau_{n} \\
& \theta_{n}=\left[\begin{array}{l}
\theta_{1} \\
\theta_{2} \\
\theta_{3}
\end{array}\right]
\end{aligned}
$$

Equations (2)-(4) are the dynamic equations of each joint. The dynamic equation of link 1 :

$$
\begin{aligned}
& \tau_{1}=\left[\begin{array}{l}
\left(m_{1} L_{1}^{2}+I_{1}\right) \\
+m_{2}\left(L_{1}^{2}+R_{C 2}^{2}+2 L_{1} R_{C 2} \cos \theta_{2}\right) \\
+m_{3}\left(\begin{array}{l}
L_{1}^{2}+L_{2}^{2}+R_{C 3}^{2}+2 L_{1} L_{2} \cos \theta_{2}+ \\
2 L_{1} R_{C 3} \cos \left(\theta_{2}+\theta_{3}\right)+2 L_{2} R_{C 3} \cos \theta_{3}
\end{array}\right) \\
+I_{2}+I_{3}
\end{array}\right) \ddot{\theta}_{1}
\end{aligned}
$$

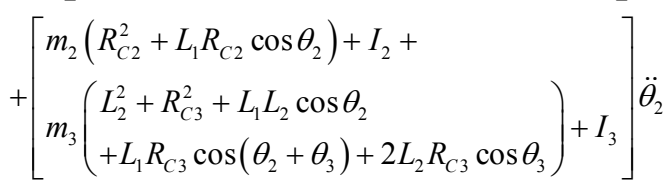

$$
\begin{aligned}
& -\left[\begin{array}{l}
\left(m_{2} L_{1} R_{C 2}+m_{3} L_{1} L_{2}\right) \sin \theta_{2} \\
+m_{2} L_{1} R_{C 3} \sin \left(\theta_{2}+\theta_{3}\right)
\end{array}\right] \dot{\theta}_{2}^{2} \\
& -\left[m_{3} L_{1} R_{C 3} \sin \left(\theta_{2}+\theta_{3}\right)+m_{3} L_{2} R_{C 3} \sin \theta_{3}\right] \dot{\theta}_{3}^{2} \\
& -\left[\begin{array}{l}
\left(2 m_{2} L_{1} R_{C 2}+2 m_{3} L_{1} L_{2}\right) \sin \theta_{2} \\
+2 m_{3} L_{1} R_{C 3} \sin \left(\theta_{2}+\theta_{3}\right)
\end{array}\right] \dot{\theta}_{1} \dot{\theta}_{2} \\
& -\left[\begin{array}{l}
\left(m_{2} L_{1} R_{C 3}+m_{3} L_{1} R_{C 3}\right) \sin \left(\theta_{2}+\theta_{3}\right) \\
+m_{3} L_{2} R_{C 3} \sin \theta_{3}
\end{array}\right] \dot{\theta}_{2} \dot{\theta}_{3} \\
& -\left[2 m_{3} L_{1} R_{C 3} \sin \left(\theta_{2}+\theta_{3}\right)+2 m_{3} L_{2} R_{C 3} \sin \theta_{3}\right] \dot{\theta}_{1} \dot{\theta}_{3} \\
& +m_{1} g R_{C 1} \cos \theta_{1} \\
& +m_{2} g\left[L_{1} \cos \theta_{1}+R_{C 2} \cos \left(\theta_{1}+\theta_{2}\right)\right] \\
& +m_{3} \cdot g\left[\begin{array}{l}
L_{1} \cos \theta_{1}+L_{2} \cos \left(\theta_{1}+\theta_{2}\right) \\
+R_{C 3} \cos \left(\theta_{1}+\theta_{2}+\theta_{3}\right)
\end{array}\right]
\end{aligned}
$$

The dynamic equation of link 2 :

$$
\tau_{2}=\left[\begin{array}{l}
m_{2}\left(R_{C 2}^{2}+L_{1} R_{C 2} \cos \theta_{2}\right)+I_{2} \\
+m_{3}\left(\begin{array}{l}
L_{2}^{2}+R_{C 3}^{2}+L_{1} L_{2} \cos \theta_{2} \\
+L_{1} R_{C 3} \cos \left(\theta_{2}+\theta_{3}\right) \\
+L_{2} R_{C 3} \cos \theta_{3}
\end{array}\right) \\
+I_{3}
\end{array}\right] \ddot{\theta}_{1}
$$




$$
\begin{aligned}
& +\left[\begin{array}{l}
m_{2} R_{C 2}^{2}+I_{2}+m_{3}\left(\begin{array}{l}
L_{2}^{2}+R_{C 3}^{2} \\
+2 L_{2} R_{C 3} \cos \theta_{3}
\end{array}\right) \ddot{\theta}_{2} \\
+I_{3}
\end{array}\right] \\
& +\left[m_{3}\left(R_{C 3}^{2}+L_{2} R_{C 3} \cos \theta_{3}\right)+I_{3}\right] \ddot{\theta}_{3} \\
& -\left[m_{3} L_{1} R_{C 3} \sin \theta_{3}\right] \dot{\theta}_{3}^{2} \\
& -\left[\begin{array}{l}
\left(m_{2} L_{1} R_{C 2}+m_{3} L_{1} L_{2}\right) \sin \theta_{2} \\
+m_{3} L_{1} R_{C 3} \sin \left(\theta_{2}+\theta_{3}\right)
\end{array}\right] \dot{\theta}_{1} \dot{\theta}_{2} \\
& -\left[\begin{array}{l}
\left.2 m_{3} L_{2} R_{C 3} \sin \theta_{3}\right] \dot{\theta}_{2} \dot{\theta}_{3} \\
-\left[\begin{array}{l}
m_{3} L_{1} R_{C 3} \sin \left(\theta_{2}+\theta_{3}\right) \\
+2 m_{3} L_{2} R_{C 3} \sin \theta_{3}
\end{array}\right] \dot{\theta}_{1} \dot{\theta}_{3}
\end{array}\right. \\
& +\left\{\begin{array}{l}
m_{2} g R_{C 2} \cos \left(\theta_{1}+\theta_{2}\right) \\
\left.+m_{3} \cdot g\left[\begin{array}{l}
L_{2} \cos \left(\theta_{1}+\theta_{2}\right)+ \\
R_{C 3} \cos \left(\theta_{1}+\theta_{2}+\theta_{3}\right)
\end{array}\right]\right\}
\end{array}\right.
\end{aligned}
$$

The dynamic equation of link 3 :

$$
\begin{aligned}
& \tau_{3}=\left[\begin{array}{l}
m_{3}\left(\begin{array}{l}
R_{C 3}^{2}+L_{1} R_{C 3} \cos \left(\theta_{2}+\theta_{3}\right)+ \\
L_{2} R_{C 3} \cos \theta_{3}
\end{array}\right) \ddot{\theta}_{1} \\
+I_{3}
\end{array}\right]+\left[m_{3}\left(R_{C 3}^{2}+L_{2} R_{C 3} \cos \theta_{3}\right)+I_{3}\right] \ddot{\theta}_{2} \\
& +\left[m_{3} R_{C 3}^{2}+I_{3}\right] \ddot{\theta}_{3} \\
& -\left[m_{3} L_{1} R_{C 3} \sin \theta_{3}\right] \dot{\theta}_{3}^{2} \\
& -\left[m_{3} L_{1} R_{C 3} \sin \left(\theta_{2}+\theta_{3}\right)\right] \dot{\theta}_{1} \dot{\theta}_{2} \\
& -\left[m_{3} L_{2} R_{C 3} \sin \theta_{3}\right] \dot{\theta}_{2} \dot{\theta}_{3} \\
& -\left[m_{3} L_{1} R_{C 3} \sin \left(\theta_{2}+\theta_{3}\right)+m_{3} L_{2} R_{C 3} \sin \theta_{3}\right] \dot{\theta}_{1} \dot{\theta}_{3} \\
& +\left\{m_{3} \cdot g \cdot R_{C 3} \cdot \cos \left(\theta_{3}+\theta_{2}+\theta_{1}\right)\right\}
\end{aligned}
$$

The general form as (1) is rewritten as follows:

$$
D_{n}(\theta) \ddot{\theta}+C_{n}(\theta, \dot{\theta})+G_{n}(\theta)=\tau_{n}
$$

where $L_{1}, L_{2}, L_{3}$ are the lengths, $C_{1}, C_{2}, C_{3}$ the centers, $R_{1}, R_{2}$, $R_{3}$ the lengths of the centers, $D_{n}$ the inertial matrix, $H_{n}$ the matrix of centrifugal and Coriolis forces, and $G_{n}$ the gravitational force matrix of the links 1,2 , and 3 respectively.

$$
\begin{aligned}
\theta_{n} & =\left[\begin{array}{lll}
\theta_{1} & \theta_{2} & \theta_{3}
\end{array}\right]^{T} \\
\dot{\theta}_{n} & =\left[\begin{array}{lll}
\dot{\theta}_{1} & \dot{\theta}_{2} & \dot{\theta}_{3}
\end{array}\right]^{T} \\
\ddot{\theta}_{n} & =\left[\begin{array}{lll}
\ddot{\theta}_{1} & \ddot{\theta}_{2} & \ddot{\theta}_{3}
\end{array}\right]^{T} \\
\tau_{n} & =\left[\begin{array}{lll}
\tau_{1} & \tau_{2} & \tau_{3}
\end{array}\right]^{T}
\end{aligned}
$$

$$
\begin{aligned}
& D_{n}=\left[\begin{array}{lll}
D_{11} & D_{12} & D_{13} \\
D_{21} & D_{22} & D_{23} \\
D_{31} & D_{32} & D_{33}
\end{array}\right] \\
& C_{n}=\left[\begin{array}{lll}
C_{11} & C_{12} & C_{13} \\
C_{21} & C_{22} & C_{23} \\
C_{31} & C_{32} & C_{33}
\end{array}\right] \\
& G_{n}=\left[\begin{array}{lll}
G_{1} & G_{2} & G_{3}
\end{array}\right]^{T}
\end{aligned}
$$

\section{SLIDING MODE CONTROLLER FOR THE RESEARCHED MODEL}

The sliding mode controller is generally utilized in industry and biomedical robots [15-16]. The controller can be planned regardless of the boundaries of the exploration model. Consequently, the sliding mode controller priorities include robustness, responsiveness, insensitiveness to parameter changes and noise, a simple physical model, and easy programming for the software driver. The block diagram that controls the lower extremity rehabilitation device based on the sliding mode controller is shown in Figure 3.

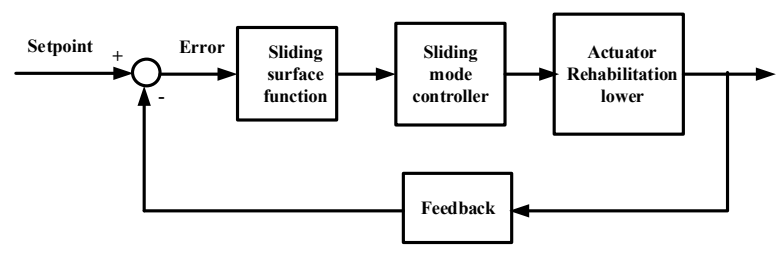

Fig. 3. Sliding mode controller block diagram.

Tracking error:

$$
e(t)=\theta_{d}(t)-\theta(t)
$$

where $\theta_{d}$ is the setpoint and $\theta$ is a reference.

Velocity and acceleration tracking error:

$$
\begin{aligned}
& \dot{e}(t)=\dot{\theta}_{d}(t)-\dot{\theta}(t) \\
& \ddot{e}(t)=\ddot{\theta}_{d}(t)-\ddot{\theta}(t)
\end{aligned}
$$

The dynamic equation (5) can be written as:

$$
\ddot{\theta}=D(q)^{-1}\{\tau-C(\theta, \dot{\theta})-G(\theta)\}
$$

Sliding surface choice function:

$$
s(t)=\lambda e(t)+\dot{e}(t)
$$

where $\lambda$ is a positive constant.

Derivative sliding surface:

$$
\dot{s}(t)=\lambda \dot{e}(t)+\ddot{e}(t)
$$

The control signal is the torque at the joints, according to [14] the control signal consists of two components which are:

$$
u=\left\{\begin{array}{lll}
u_{e q} & \text { when } & s(\theta, t)=0 \\
u_{N} & \text { when } & s(\theta, t) \neq 0
\end{array}\right.
$$


We can calculate the control signal from the differential condition $s(t)=0$ and from (10) and (8).

$$
\begin{aligned}
& \dot{s}(t)=\lambda \dot{e}(t)+\ddot{e}(t)=0 \\
& \dot{s}(t)=\lambda \dot{e}(t)+\ddot{\theta}_{d}(t)-\ddot{\theta}(t) \\
& \dot{s}(t)=\lambda \dot{e}(t)+\ddot{\theta}_{d}(t)-D(\theta)^{-1}\{\tau-C(\theta, \dot{\theta})-G(\theta)\} \\
& \dot{s}(t)=\lambda \dot{e}(t)+\ddot{\theta}_{d}(t)-D(\theta)^{-1}\left\{u_{e q}-C(\theta, \dot{\theta})-G(\theta)\right\} \\
& \dot{s}(t)=\lambda \dot{e}(t)+\ddot{\theta}_{d}(t)-D(\theta)^{-1}\left\{u_{e q}-C(\theta, \dot{\theta})-G(\theta)\right\}=0 \\
& u_{e q}=\lambda \dot{e}(t)+\ddot{\theta}_{d}(t)+D(\theta)^{-1} C(\theta, \dot{\theta})+D(\theta)^{-1} G(\theta)
\end{aligned}
$$

with $u_{N}=K \operatorname{sgn}(s)$. The sliding mode control for each joint is shown as:

$$
\begin{aligned}
& u=u_{N}+u_{e q}=\lambda \dot{e}(t)+\ddot{\theta}_{d}(t)+D(\theta)^{-1} C(\theta, \dot{\theta}) \\
& +D(\theta)^{-1} G(\theta)+K \operatorname{sgn}(s)
\end{aligned}
$$

We use the Lyapunov method to check the stability of the sliding mode controller. The Lyapunov function associated with the research model is:

$$
\begin{aligned}
& V(s)=\frac{1}{2} s^{2} \\
& \dot{V}(s)=\dot{s} s \\
& \dot{V}(s)=-K \operatorname{sgn}(s)
\end{aligned}
$$

For the controller to be stable we must choose a positive $K$ to satisfy the stability condition:

$$
\dot{V}(s)=-K \operatorname{sgn}(s)<0
$$

\section{SimULATION IN MATLAB}

We utilized Matlab to simulate the response of the research model when using the sliding mode controller. The parameters of the research model are shown in Table I. Simulations for the research model were conducted for 2 cases, namely without and with a torque of load acting on the joints of the research model. The position of the initial joints of the research model is:

$$
\left\{\begin{array}{c}
\theta_{1}=0^{\circ} \\
\theta_{2}=-90^{\circ} \\
\theta_{3}=90^{\circ}
\end{array}\right.
$$

The setpoint angle for the joint simulates the range of motion of the human lower limb joints.

$$
\left\{\begin{array}{l}
\theta_{1_{-} \text {setpoint }}=45^{\circ} \sin (2 * \pi * 0,1 * t)+45^{\circ} \\
\theta_{2_{-} \text {setpoint }}=-\frac{135^{0}}{2} \sin (2 * \pi * 0,1 * t)-\frac{135^{0}}{2} \\
\theta_{3_{-} \text {setpoint }}=40^{\circ} \sin (2 * \pi * 0,1 * t)+80^{\circ}
\end{array}\right.
$$

The simulation results of the case without load are shown in Figures 4-6 and with load in Figures 7-9.
TABLE I. SIMULATION PARAMETERS OF THE RESEARCH MODEL

\begin{tabular}{|c|l|c|c|}
\hline \multirow{2}{*}{} & \multicolumn{3}{|c|}{ Simulator parameters } \\
\cline { 2 - 4 } & \multicolumn{1}{|c|}{ Description } & Value & Parameter \\
\hline 1 & Mass of link 1 & 5 & $m_{1}(\mathrm{~kg})$ \\
\hline 2 & Mass of link 2 & 5 & $m_{2}(\mathrm{~kg})$ \\
\hline 3 & Mass of link 3 & 1 & $m_{3}(\mathrm{~kg})$ \\
\hline 4 & Length of link 1 & 0.4 & $L_{1}(\mathrm{~m})$ \\
\hline 5 & Length of link 2 & 0.45 & $L_{2}(\mathrm{~m})$ \\
\hline 6 & Length of link 3 & 0.2 & $L_{3}(\mathrm{~m})$ \\
\hline 7 & Length center of link 1 & $R_{c l}(\mathrm{~m})$ \\
\hline 8 & Length center of link 2 & 0.225 & $R_{c 2}(\mathrm{~m})$ \\
\hline 9 & Length center of link 3 & 0.1 & $R_{c 3}(\mathrm{~m})$ \\
\hline 10 & Moment of inertia of link 1 & 0.2667 & $I_{1}\left(\mathrm{~kg} \cdot \mathrm{m}^{2}\right)$ \\
\hline 11 & Moment of inertia of link 2 & 0.3375 & $I_{2}\left(\mathrm{~kg} . \mathrm{m}^{2}\right)$ \\
\hline 12 & Moment of inertia of link 3 & 0.0133 & $I_{3}\left(\mathrm{~kg} . \mathrm{m}^{2}\right)$ \\
\hline 13 & Cofficient $K$ & 40 & \\
\hline 14 & Cofficient $\lambda$ & 20 & \\
\hline 15 & Load Torque on link 1 & 50 & $(\mathrm{~N} . \mathrm{m})$ \\
\hline 16 & Load Torque on link 2 & 50 & $(\mathrm{~N} . \mathrm{m})$ \\
\hline 17 & Load Torque on link 3 & 10 & $(\mathrm{~N} . \mathrm{m})$ \\
\hline
\end{tabular}
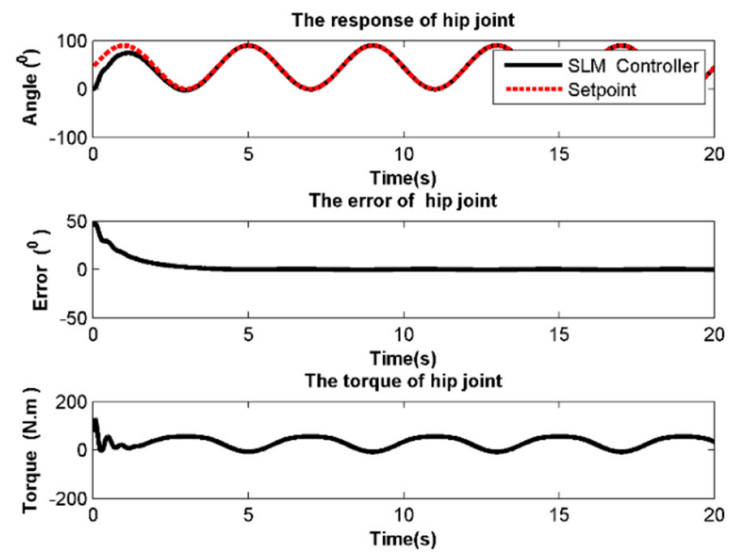

Fig. 4. Response of link 1without load.
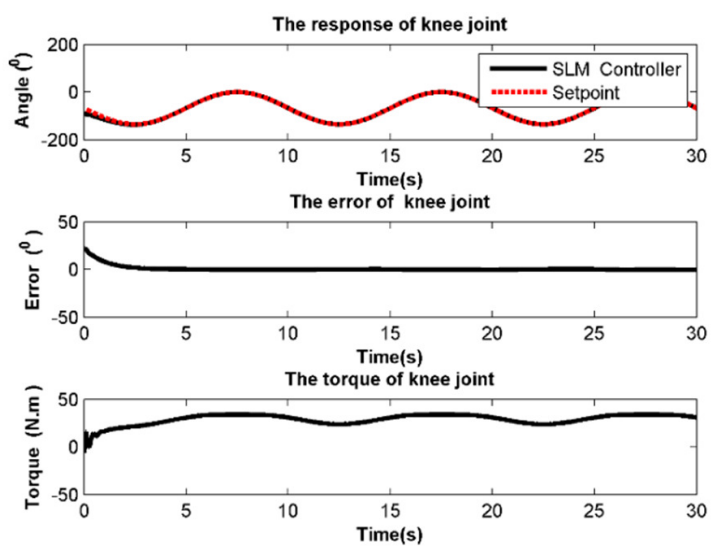

Fig. 5. Response of link 2 without load.

Figures 4-6 present the response of the research model's hip, knee, and ankle joints when using the sliding mode controller without load. The hip joint results are: settling time $=$ $1 \mathrm{~s}$, maximum torque $=150 \mathrm{Nm}$, and overshoot $=0$. The knee joint results are: settling time $=0.5 \mathrm{~s}$, maximum torque $=$ $30 \mathrm{Nm}$, and overshoot $=0$. The ankle joint results are: settling time $=0.5 \mathrm{~s}$, maximum torque $=6 \mathrm{Nm}$, and overshoot $=0$. 

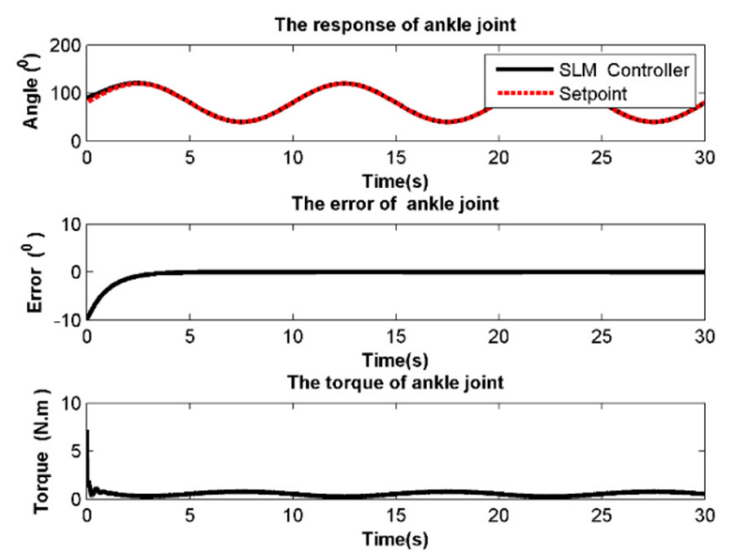

Fig. 6. Response of link 3 without load.

The sliding mode controller results for the case with load are shown in Figures 7-9. The hip joint results are: settling time $=1 \mathrm{~s}$, maximum torque $=80 \mathrm{Nm}$, and overshoot $=0$. The knee joint results are: settling time $=0.5 \mathrm{~s}$, maximum torque $=$ $31 \mathrm{Nm}$, and overshoot $=0$. The results of the ankle joint are: the settling time $=0.5 \mathrm{~s}$, maximum torque $=6 \mathrm{Nm}$, and overshoot $=$ 0 .
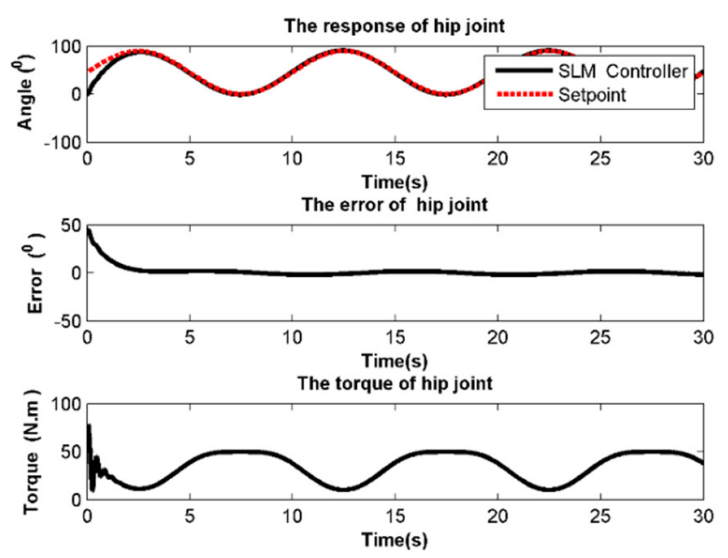

Fig. 7. Response of link 1 with load.
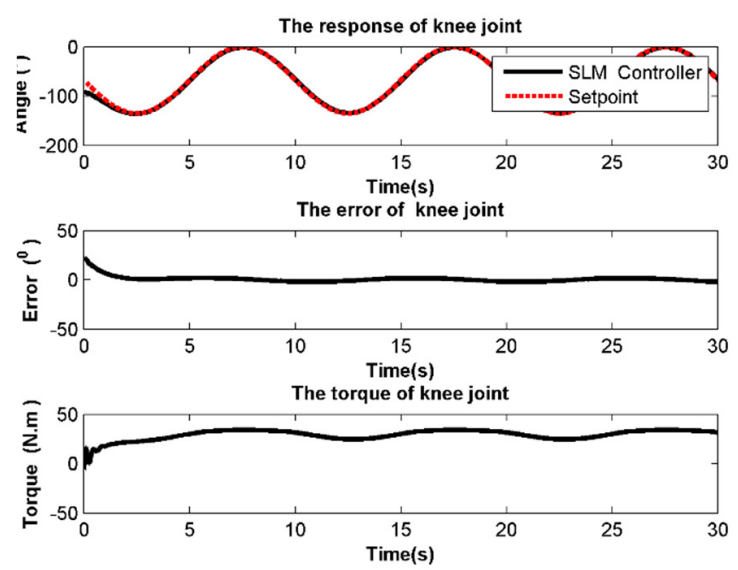

Fig. 8. Response of link 2 with load.
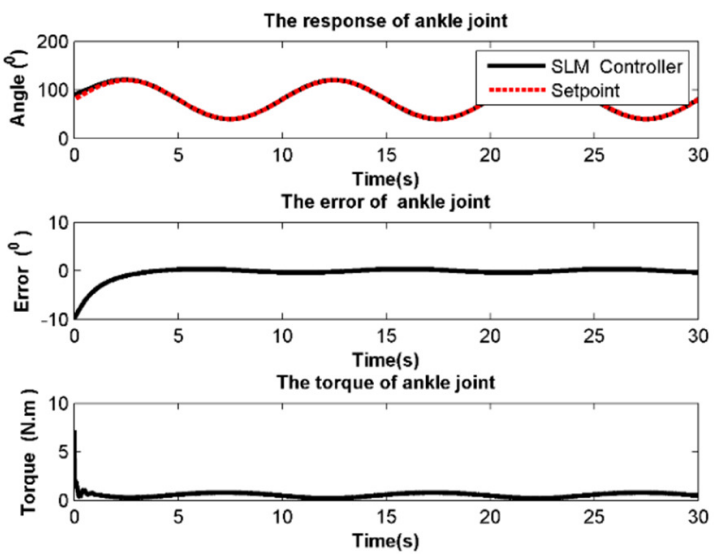

Fig. 9. Response of link 3 with load.

\section{DISCUSSION}

In this study, a sliding controller for a lower extremity rehabilitation exercise aid was designed and its response was simulated in Matlab. The simulation results of the device in the flexion/extension movements of the hip, knee, and ankle joints are shown in Figures 4-9. The hip joint results are shown in Figures 4 and 7 , which show that this is the joint that bears the most significant internal load due to the influence of the weight of the structure and the external force, thus creating the most torque. For the knee joint, the results are shown in Figures 5 and 8. The joint can only bear the internal load of the mechanism and the external force, so the maximum torque is 31 N.m. Finally, the results of the ankle joint are shown in Figures 6 and 9. This is the joint that only bears the internal load of the mechanism and the minor external force, so the torque is minimal. In addition, in order to assess the adequacy of the proposed controller, the comparison of the trajectory tracking of the proposed controller with several controllers studied in [17-19] was conducted. The trajectory error tracking of the study compared with the works in [17-19] is similar, however, the response time in this study was better $(1 \mathrm{~s}$ instead of $2 \mathrm{~s}$ ). In addition, the above studies have not considered the influence of external [17] or small [18-19] loads on the research model and have not analyzed the action moments of each joint. Therefore, this study demonstrates that the sliding controller can control the lower extremity rehabilitation device for patients with fast response time and slight error. The advantage of this study is that we have created a controller that is not affected by external forces, which makes the device response stable, which is essential in the rehabilitation setting for patients. The main limitation of the current study is that it only simulates the response, so it is not possible to conclude that this is the actual response when the controller is applied to real devices. The development direction of this research is to check the correctness of the controller when applied on real devices.

\section{CONCLUSION}

A sliding controller that controls a 3 DoF robot model that supports lower extremity rehabilitation was presented in this paper. The dynamic model of the research model was studied. It is proposed to apply the sliding mode controller to study the 
dynamic response of the links of the research model. The study results have been verified in Matlab simulations of the response of the links of the research model. The proposed sliding mode controller for the research model has shown a good response with small error tracking and torque compared to other studies. This result is the basis for manufacturing an actual device to support lower extremity rehabilitation for stroke patients using a linear actuator.

\section{ACKNOWLEDGMENT}

This work is funded by the Pham Van Dong University, Vietnam, project $13 / 2017 / \mathrm{KHCN}$. The authors are grateful for the financial support.

\section{REFERENCES}

[1] E. Berge and P. Sandercock, "Specific treatment of acute ischemic stroke," in Warlow's Stroke, John Wiley \& Sons, Ltd, 2019, pp. 587656, https://doi.org/10.1002/9781118492390.ch13.

[2] L. V. Thanh, "Network of care for stroke, the past and the future", Journal of Clinical Medicine, Vol. 10, pp. 11-14, 2015.

[3] D. Shi, W. Zhang, W. Zhang, and X. Ding, "A Review on Lower Limb Rehabilitation Exoskeleton Robots," Chinese Journal of Mechanical Engineering, vol. 32, no. 1, Aug. 2019, Art. no. 74, https://doi.org/ 10.1186/s10033-019-0389-8.

[4] M. Rahmani and M. H. Rahman, "Novel robust control of a 7-DOF exoskeleton robot," PLOS ONE, vol. 13, no. 9, 2018, Art. no. e0203440, https://doi.org/10.1371/journal.pone.0203440.

[5] M. Torabi, M. Sharifi, and G. Vossoughi, "Robust adaptive sliding mode admittance control of exoskeleton rehabilitation robots," Scientia Iranica, vol. 25, no. 5, pp. 2628-2642, Oct. 2018, https://doi.org/ $10.24200 /$ sci.2017.4512

[6] S. Zhu, X. Jin, B. Yao, Q. Chen, X. Pei, and Z. Pan, "Non-linear sliding mode control of the lower extremity exoskeleton based on human-robot cooperation," International Journal of Advanced Robotic Systems, vol. 13, no. 5, Sep. 2016, Art. no. 1729881416662788, https://doi.org/ 10.1177/1729881416662788.

[7] Y. Tu et al., "An Adaptive Sliding Mode Variable Admittance Control Method for Lower Limb Rehabilitation Exoskeleton Robot," Applied Sciences, vol. 10, no. 7, Jan. 2020, Art. no. 2536, https://doi.org/ 10.3390/app 10072536

[8] S. Babesse, "Design of Two Optimized Controllers of a Hydraulic Actuator Semi-Active Suspension: A Comparison Study," Engineering, Technology \& Applied Science Research, vol. 9, no. 4, pp. 4561-4565, Aug. 2019, https://doi.org/10.48084/etasr.2836.

[9] M. K. Joyo et al., "Optimized Proportional-Integral-Derivative Controller for Upper Limb Rehabilitation Robot," Electronics, vol. 8, no. 8, Aug. 2019, Art. no. 826, https://doi.org/10.3390/electronics 8080826.

[10] H. Medjoubi, A. Yassine, and H. Abdelouahab, "Design and Study of an Adaptive Fuzzy Logic-Based Controller for Wheeled Mobile Robots Implemented in the Leader-Follower Formation Approach," Engineering, Technology \& Applied Science Research, vol. 11, no. 2, pp. 6935-6942, Apr. 2021, https://doi.org/10.48084/etasr.3950.

[11] W. Huang, H. Xu, X. Fan, and Y. Lin, "Control of Lower Limb Rehabilitation Robot Based on Fuzzy PID," in 2018 3rd International Conference on Control, Automation and Artificial Intelligence (CAAI 2018), Aug. 2018, pp. 6-8, https://doi.org/10.2991/caai-18.2018.2.

[12] G. Al Rezage and M. O. Tokhi, "Fuzzy PID control of lower limb exoskeleton for elderly mobility," in 2016 IEEE International Conference on Automation, Quality and Testing, Robotics (AQTR), ClujNapoca, Romania, May 2016, https://doi.org/10.1109/AQTR.2016. 7501310.

[13] D. Jiang, G. Shi, Z. Pang, S. Li, and Y. Tian, "Control of a New Cycling Rehabilitation Robot Based on Fuzzy PID," Journal of Physics: Conference Series, vol. 1622, Sep. 2020, Art. no. 012119, https://doi.org/10.1088/1742-6596/1622/1/012119.
[14] Z. Chen, Q. Guo, Y. Yan, and D. Jiang, "Robust Sliding Mode Control for a 2-DOF Lower Limb Exoskeleton Base on Linear Extended State Observer," Mechanical Engineering Science, vol. 2, no. 2, Dec. 2020, https://doi.org/10.33142/mes.v2i2.3160.

[15] J. Liu, Y. Zhang, J. Wang, and W. Chen, "Adaptive sliding mode control for a lower-limb exoskeleton rehabilitation robot," in 2018 13th IEEE Conference on Industrial Electronics and Applications (ICIEA), Wuhan, China, May 2018, pp. 1481-1486, https://doi.org/10.1109/ICIEA. 2018.8397943.

[16] B. Maalej, H. Medhaffar, A. Chemori, and N. Derbel, "A Fuzzy Sliding Mode Controller for Reducing Torques Applied to a Rehabilitation Robot," in 2020 17th International Multi-Conference on Systems, Signals Devices (SSD), Monastir, Tunisia, Jul. 2020, pp. 740-746, https://doi.org/10.1109/SSD49366.2020.9364130.

[17] T. B. Trung, G. L. T. Hong, Q. D. Hai, and N. P. V. Bach, "Advanced Method for Motion Control of a 3 DOFs Lower Limb Rehabilitation Robot," International Journal of Innovative Technology and Interdisciplinary Sciences, vol. 2, no. 4, pp. 316-325, Nov. 2019, https://doi.org/10.15157/IJITIS.2019.2.4.316-325.

[18] Z. Shen, J. Zhou, J. Gao, and R. Song, "Fuzzy Logic Based PID Control of a 3 DOF Lower Limb Rehabilitation Robot," in 2018 IEEE 8th Annual International Conference on CYBER Technology in Automation, Control, and Intelligent Systems (CYBER), Tianjin, China, Jul. 2018, pp. 818-821, https://doi.org/10.1109/CYBER.2018.8688089.

[19] D. T. Hai and D. T. Vu, "An Intelligent Control for Lower Limb Exoskeleton for Rehabilitation," International Journal of Electrical and Electronics Engineering, vol. 4, no. 8, pp. 13-19, 2017, https://doi.org/ 10.14445/23488379/IJEEE-V4I8P 103. 